\title{
Prioritized Contention Resolution for Random Access Networked Control Systems
}

\author{
Mohammad H. Mamduhi ${ }^{1,3}$, Mikhail Vilgelm² ${ }^{2}$ Wolfgang Kellerer ${ }^{2}$, and Sandra Hirche ${ }^{1}$
}

\begin{abstract}
Decentralized scheduling schemes offer low-cost, easy-to-install, and scalable resource allocation mechanisms suitable for networked control systems (NCS) with a large number of loops. However, contentions are unavoidable as collisions randomly occur in the communication link which lowers channel throughput and consequently compromises control performance. In this paper, we introduce a novel deterministic state-dependent prioritization policy to resolve the contention in a multi-loop NCS with random access medium. The proposed prioritization scheme can be realized within most of the shared medium technologies, e.g. ad-hoc and bus networks, aiming to decrease the collision probability. The priority for an individual control loop depends on the current local network-induced errors with a larger error leading to higher priority. We show stability of the described NCS under the proposed collision reduction mechanism according to stochastic Lyapunovbased techniques. It is demonstrated that the proposed statedependent contention resolution is considerably effective in lowering the collision rate, though incapable of eliminating it due to the decentralized nature of the medium access control.
\end{abstract}

\section{INTRODUCTION}

Smart grids and autonomous vehicular systems are application examples of networked control systems with multiple feedback control loops exchanging data over a shared communication infrastructure. This calls for the advent of more intelligent control and communication schemes to support the real-time requirements of such coupled systems [1]. This is often extremely challenging due to the compromising impact of the shared medium such as capacity constraints, contention, data loss, and latency [2]. The mentioned phenomena impair the communication quality and control performance and may even lead the overall NCS to instability. Communication systems are more often designed in static fashion, i.e., neither the real-time situation of sending/receiving stations nor channel status are taken into account in resource allocation. Carrier sense multiple access (CSMA), time/frequency division multiple access (TDMA-FDMA), and (un-)slotted ALOHA are well-known static channel access mechanisms. There has been an increased attention recently in the joint design of control and communication schemes in NCSs in

\footnotetext{
${ }^{1}$ M. H. Mamduhi, and S. Hirche are with the Chair of InformationOriented Control, Technical University of Munich, Arcisstraße 21, D-80290 München, Germany http://www.itr.ei.tum.de, \{mh.mamduhi, hirche\}@tum.de

${ }^{2}$ M. Vilgelm, and W. Kellerer are with the Chair of Communication Networks, Technical University of Munich, Arcisstraße 21, D-80290 München, Germany http://www.lkn.ei.tum.de, \{mikhail.vilgelm, wolfgang.kellerer\}etum.de

${ }^{3}$ M. H. Mamduhi is with the Department of Automatic Control, The Royal Institute of Technology, SE-100 44 Stockholm, Sweden, mamduhi@kth.se
}

order to increase control performance and communication quality. It is shown in recent works that state-dependent control and scheduling approaches often outperform static schemes in terms of control performance and consuming significantly less of the often-costly resource [3]-[5].

Within the context of control-aware communication design in NCSs, real-time prioritization can be effectively implemented by awarding limited resources to systems with the most critical control conditions. Typically, those conditions are formulated as functions of control-related or channelrelated states. Deterministic transmission conditions often appear as event-triggers such as threshold policies [5][7]. Prioritization is alternatively employed in probabilistic fashion through assigning transmission probabilities to each sending station according to state-dependent priority measures [8]-[10]. It is shown that probabilistic prioritization can be employed for NCSs with random access channels resulting in an improved performance compared to non-prioritized counterparts [11]. Try-once-discard (TOD) is one of the well-known deterministic prioritized resource assignment mechanisms [7]. Based on current measurement data, TOD prioritizes transmissions by awarding the channel access to the systems with the largest discrepancy between the true and estimated state values. However, prioritization in the original TOD formulation can be realized at the expense of having a centralized coordination unit, which is not often desired in large NCSs due to scalabality issues. Moreover, centralized coordination of medium access is not possible in many scenarios, such as establishing a network connection, which is performed in a decentralized fashion [12], [13]. Connection establishment is envisioned to be the dominating part of the end-to-end delay for future NCS applications, and fast random access is one of the challenges for the evolution of wireless networks towards 5G [12], [14].

In this paper, we introduce a scalable dynamic prioritizing channel access mechanism for NCSs by employing a binary countdown technique, which is a well-accepted contention resolution strategy in most of the shared medium technologies: wireless, bus or powerline communication networks [15], [16]. We apply it to the random access problem, where all sub-systems access the medium in a decentralized fashion. Stability of NCSs under the proposed scheme is shown employing Lyapunov-based concept of stochastic stability. Numerical analyses demonstrate a considerable performance improvement compared to the state-of-the-art decentralized techniques. It is shown that the proposed scheme renders lower collision rate compared with the other common scheduling protocols, if a large number of systems 


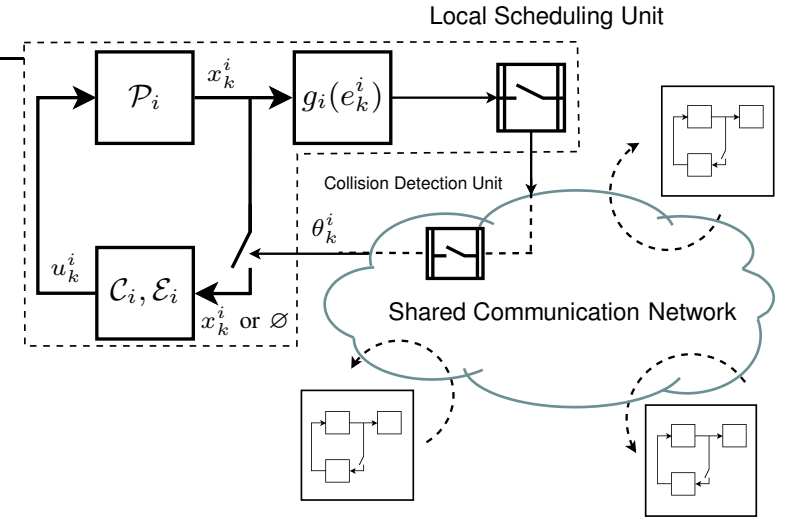

Fig. 1: Schematic of a shared resource multi-loop NCS with local scheduling unit.

utilize the communication network.

As the remainder of this paper, we present the problem formulation in Section II. The priority assignment mechanism for random access channels is introduced in Section III. Stability properties of the described NCS under the proposed channel arbitration is discussed in Section IV. Simulation results and discussions are followed in Section V.

Notation: In this paper we denote the Euclidean norm, and conditional expectation by $\|\cdot\|_{2}$, and $\mathrm{E}[\cdot \cdot \cdot]$, respectively. The trace and ceiling operators are represented by $\operatorname{tr}(\cdot)$ and $\lceil\cdot\rceil$, respectively. A random vector $X$ from a multivariate Gaussian distribution with mean vector $\mu$ and covariance matrix $\Sigma$ is denoted by $X \sim \mathcal{N}(\mu, \Sigma)$. A vector's superscript indicates the belonging sub-system and its subscript denotes the time instance. For matrices, subscripts indicate the belonging subsystems, and superscripts represent the matrix power.

\section{PROBLEM STATEMENT}

We consider NCSs consisting of $N$ heterogeneous linear controlled sub-systems that share a communication medium with capacity limitations. Local sub-systems $i \in\{1, \ldots, N\}$ consist of an LTI stochastic process $\mathcal{P}_{i}$, and a control unit including a state estimator $\mathcal{E}_{i}$ and a feedback controller $\mathcal{C}_{i}$, see Fig. 1. Local state information of a sub-system pass through the shared channel to be received by its corresponding control unit. Each sub-system $i$ is modeled in discretetime by the following stochastic state-space equation

$$
x_{k+1}^{i}=A_{i} x_{k}^{i}+B_{i} u_{k}^{i}+w_{k}^{i}
$$

with $x_{k}^{i} \in \mathbb{R}^{n_{i}}$ and $u_{k}^{i} \in \mathbb{R}^{m_{i}}$ denoting local system state, and control input of sub-system $i$, respectively, and $A_{i} \in \mathbb{R}^{n_{i} \times n_{i}}$, and $B_{i} \in \mathbb{R}^{n_{i} \times m_{i}}$ describing system matrix, and input matrix. We assume each pair $\left(A_{i}, B_{i}\right)$ to be controllable. The system noise is assumed to be a random sequence with independent and identically distributed (i.i.d.) realizations $w_{k}^{i} \sim \mathcal{N}\left(0, W_{i}\right)$. The initial state $x_{0}^{i}$ is randomly chosen from an arbitrary finite-variance distribution, for $i \in\{1, \ldots, N\}$.

Due to limited capacity of the communication channel, only a limited number of sub-systems can transmit simultaneously. To determine feasibility of a transmission, decentralized scheduling units are integrated within each sub-system. At every time-step $k$, the variable $\delta_{k}^{i} \in\{0,1\}$ denotes either "transmit" or "backoff" for sub-systems $i$ as follows

$$
\delta_{k}^{i}= \begin{cases}1, & x_{k}^{i} \text { sent through the channel } \\ 0, & x_{k}^{i} \text { blocked. }\end{cases}
$$

We assume that the transmitted packets are not lost due to any external cause (see [17] where the problem of data packet loss in a similar NCS setup is addressed). Hence, if data packets do not collide in the channel, they will certainly be received by their corresponding control units. We assume that if a collision occurs in the communication channel it will be detected, then involved sub-systems are blocked and data packets are dropped. We define the binary variable $\gamma_{k}^{i}$ as the collision indicator at a time-step $k$ as follows

$$
\gamma_{k}^{i}= \begin{cases}1, & x_{k}^{i} \text { successfully received } \\ 0, & x_{k}^{i} \text { collided }\end{cases}
$$

Upon receiving new state information by the control unit, the control input is computed by a local feedback controller. In case of a non-transmission, a model-based estimation of the system state is employed. Defining the information history at the controller $\mathcal{C}_{i}$ at a time-step $k$ as $\mathcal{I}_{k}^{i}=$ $\left\{\theta_{0}^{i}, x_{0}^{i}, \ldots, \theta_{k-1}^{i}, x_{k-1}^{i}\right\}$, where $\theta_{k}^{i} \triangleq \delta_{k}^{i} \gamma_{k}^{i}$, we have

$$
\mathrm{E}\left[x_{k}^{i} \mid \mathcal{I}_{k}^{i}\right]= \begin{cases}x_{k}^{i}, & \theta_{k}^{i}=1, \\ \left(A_{i}-B_{i} L_{i}\right) \mathrm{E}\left[x_{k-1}^{i} \mid \mathcal{I}_{k-1}^{i}\right], & \theta_{k}^{i}=0 .\end{cases}
$$

Linear state-feedback control $u_{k}^{i}$ is computed according to the following measurable and causal mapping of $\mathcal{I}_{k}^{i}$ :

$$
u_{k}^{i}=-L_{i} \mathrm{E}\left[x_{k}^{i} \mid \mathcal{I}_{k}^{i}\right],
$$

where, $L_{i} \in \mathbb{R}^{m_{i} \times n_{i}}$ is a stabilizing feedback control gain. According to (3), the control inputs are realized with true state value $x_{k}^{i}$ if transmission is successful. Otherwise, the control inputs are computed with model-based estimate $\mathrm{E}\left[x_{k}^{i}\right]$. The estimate (2) is well-behaved since the control gain $L_{i}$ is stabilizing, and the pair $\left(A_{i}, B_{i}\right)$ is stabilizable.

We introduce the network-induced error $e_{k}^{i}$ for each subsystem $i \in\{1, \ldots, N\}$, at every time-step $k$, as follows

$$
e_{k}^{i} \triangleq x_{k}^{i}-\mathrm{E}\left[x_{k}^{i} \mid \mathcal{I}_{k}^{i}\right]
$$

Consider the concatenating vector $\left[x_{k}^{i^{\top}} e_{k}^{i^{\top}}\right]^{\top}$ as the aggregate state of sub-system $i$ in the NCS. It is then straightforward to derive the following local dynamics according to (1)-(4)

$$
\begin{aligned}
x_{k+1}^{i} & =\left(A_{i}-B_{i} L_{i}\right) x_{k}^{i}+B_{i} L_{i} e_{k}^{i}+w_{k}^{i}, \\
e_{k+1}^{i} & =\left(1-\theta_{k}^{i}\right) A_{i} e_{k}^{i}+w_{k}^{i} .
\end{aligned}
$$

It can be seen from (6) that evolution of error state $e_{k}^{i}$ is independent from the system state $x_{k}^{i}$ within every local sub-system. This enables us to choose a stabilizing controller a priori as in (3). Note that stabilizability of the pair $\left(A_{i}, B_{i}\right)$ ensures that the closed-loop matrix $\left(A_{i}-B_{i} L_{i}\right)$ is stable. Hence, it follows from (5) that sub-system $i$ with the aggregate state $\left[x_{k}^{i^{\top}} e_{k}^{i^{\top}}\right]^{\top}$ is stable if the error state converges. 


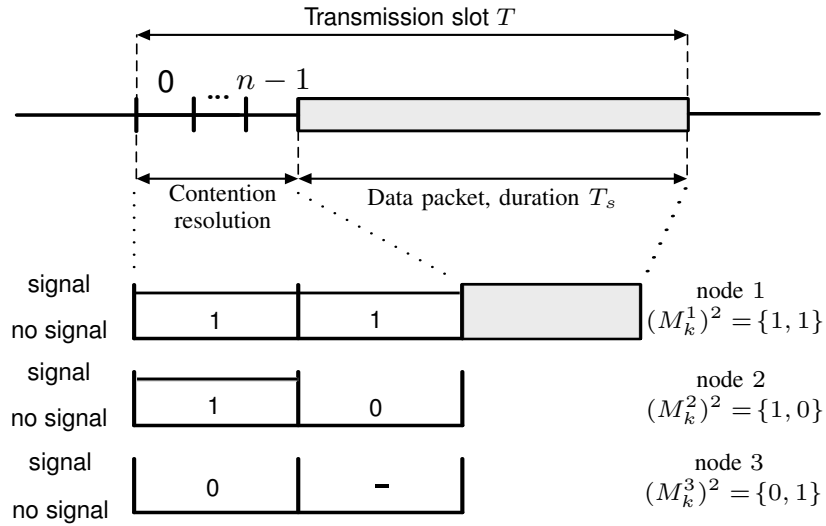

Fig. 2: Transmission slot structure and priority resolution example.

\section{Priority-BAsed Contention Resolution MAC}

We assume that the communication protocol is based on a time slotted medium access (there is a basic level of coarse synchronization between the nodes, easily achieved with periodic broadcast signals [18]), where the timeline is divided into transmission slots of equal duration $T$, where $T$ is the sampling period of control systems. Conventional random access protocols assume that all communicating nodes send the data directly in the closest available transmission slot, so if at least another node sends in the same slot, transmissions collide and data packets are lost. The communication channel is assumed to be under the following per-time-step constraint

$$
\sum_{i=1}^{N} \theta_{k}^{i} \leq 1
$$

which specifies that at most one node successfully transmits at a time-step. Here, we consider the random access protocol with priority-based contention resolution [16], [18]. Compared to the non-prioritized random access schemes, the modified transmission slot structure consists of the contention resolution period of duration $n T_{\mathrm{CR}}$, and data transmission period of duration $T_{s}$, see Fig. 2. Contention resolution period consists of $n$ slots, each of duration $T_{\mathrm{CR}}$. Prior to a transmission at a time-step $k$, a node $i$ is assigned with a priority level $\hat{m}_{k}^{i} \in \mathcal{K} \in \mathbb{N}_{0}$, where $\mathcal{K}$ is a common finite set of priorities. The value of $\hat{m}_{k}^{i}$ is mapped onto a binary sequence $\left(M_{k}^{i}\right)^{n}=\left\{m_{k}^{i, 0}, m_{k}^{i, 1}, \ldots, m_{k}^{i, j}, \ldots, m_{k}^{i, n-1}\right\}$ with length $n$ (padded with zeros until $n$, if necessary) as

$$
\begin{aligned}
\hat{m}_{k}^{i} & =m_{k}^{i, 0} \cdot 2^{n-1}+m_{k}^{i, 1} \cdot 2^{n-2}+\ldots \\
& +m_{k}^{i, j} \cdot 2^{n-j-1}+\ldots m_{k}^{i, n-1} \cdot 2^{0} .
\end{aligned}
$$

Having this sequence, if $m_{k}^{i, j}=1$, the node sends in the $j^{\text {th }}$ contention resolution slot of the $k^{\text {th }}$ time-step, otherwise, the node keeps listening to the medium. In case node $i$ detects the presence of a signal while listening to the $j^{\text {th }}$ slot, which means there exits a contending node $p$ with $\hat{m}_{k}^{p}>\hat{m}_{k}^{i}$, node $i$ backs off and does not send data in the current time slot.

Illustrative example: Three sub-systems (nodes) with IDs $i \in\{1,2,3\}$ are assigned with the priorities $\hat{m}_{k}^{1}=3, \hat{m}_{k}^{2}=2$ and $\hat{m}_{k}^{3}=1$, respectively, where higher value implies higher priority, see Fig. 2. Given $\mathcal{K}=\{0,1,2,3\}$, there are $n=$ $\log _{2}|\mathcal{K}|=2$ contention resolution slots. Thus, the binary priority sequences are $\left(M_{k}^{1}\right)^{2}=\{1,1\},\left(M_{k}^{2}\right)^{2}=\{1,0\}$, and $\left(M_{k}^{3}\right)^{2}=\{0,1\}$, respectively. Therefore, only node 1 and node 2 are sending signals in slot 0 (i.e., $m_{k}^{1,0}=m_{k}^{2,0}=1$ ), while node 3 is only listening to the medium $\left(m_{k}^{3,0}=0\right)$. Since node 3 detects a non-empty signal in the resolution slot 0 , it concludes that nodes with higher priorities exist, and hence backs off. At the resolution slot 1 , only node 1 sends the signal, and node 2 is detecting it while listening. Hence, node 2 does not proceed with transmission and as a result, only node 1 continues with sending its data packet.

The proposed scheme is incapable of fully resolving the contentions. If there exist at least two nodes on the highest priority in a given slot, they collide. To reduce the probability of collision, we introduce a barring factor $p_{b} \in[0,1)$ which denotes the back-off probability of each node, i.e. $\mathrm{P}\left[\delta_{k}^{i}=\right.$ $1]=1-p_{b}$. If two nodes are assigned with the highest priority $\hat{m}_{\max }$, the probability of collision is given by $\left(1-p_{b}\right)^{2}$, otherwise, if $p_{b}=0$, collision probability is 1 . The barring factor has to be carefully chosen, or dynamically adjusted, to minimize possible underutilization effects. Careful design of $p_{b}$ is a well-studied topic, see e.g. [13], [19], but is out of scope of this work. Therefore, the probability that a subsystem $i$ successfully transmits at a given time-step $k$ is

$$
\mathrm{P}\left[\theta_{k}^{i}=1\right]=\left(1-p_{b}\right) \mathrm{P}\left[\hat{m}_{k}^{i}>\hat{m}_{k}^{j}, \forall j \in\{1, \ldots, N\} \backslash\{i\}\right] .
$$

\section{A. State-dependent Priority Measure}

To determine the transmission priority of a given node, we propose a real-time deterministic mapping, which operates decentrally at every time-step $k$ for every node $i$, as follows

$$
\hat{m}_{k}^{i}= \begin{cases}0 & \text { if } g_{i}\left(e_{k}^{i}\right)<\lambda_{i}, \\ \left\lceil g_{i}\left(e_{k}^{i}\right)\right\rceil & \text { if } \lambda_{i} \leq g_{i}\left(e_{k}^{i}\right) \leq \hat{m}_{\max }, \\ \hat{m}_{\max } & \text { otherwise, }\end{cases}
$$

where $\lambda_{i}$ 's are given local constant thresholds, and $g_{i}\left(e_{k}^{i}\right)$ : $\mathbb{R}^{n_{i}} \rightarrow \mathbb{R}^{+}$is assumed to be continuous and strictly increasing with respect to $\mathcal{L}_{1}$ norm of $e_{k}^{i}$. The exact form of $g_{i}\left(e_{k}^{i}\right)$ can be determined empirically and adjusted according to the target set-up. The following results however are generic for any function $g_{i}$ 's with the mentioned characteristics. Here, $\hat{m}_{\max }$ can be dimensioned according to the number of subsystems in the network. Moreover, the required number of contention resolution slots to accommodate the priority levels up to $\hat{m}_{\max }$ can be derived as $n=\left\lceil\log _{2}\left(\hat{m}_{\max }+1\right)\right\rceil$.

\section{B. Collision Probability}

According to the priority assignment law (10), the probability that a forwarded transmission collides equals the probability that at least one more sub-system is assigned with the same highest priority at that time. Considering $p_{b}=0$, the probability that at time-step $k$, a collision occurs is

$$
\begin{aligned}
& \mathrm{P}\left[\theta_{k}^{i}=0, \forall i\right]=\mathrm{P}\left[\hat{m}_{k}^{i}=0, \forall i \in\{1, \ldots N\}\right] \\
& +\mathrm{P}[\underbrace{\hat{m}_{k}^{1}=\ldots=\hat{m}_{k}^{p}}_{\cup p \in\{2,3, \ldots, N\}}=\bar{m}_{k}>\hat{m}_{k}^{l}, l \neq p] \\
& +\mathrm{P}[\underbrace{\hat{m}_{k}^{1}=\ldots=\hat{m}_{k}^{p}}_{\cup p \in\{2,3, \ldots, N\}}=\hat{m}_{\max }],
\end{aligned}
$$


where $\bar{m}_{k}$ is an arbitrary priority assignment from the set of priorities $\mathcal{K}$, except 0 and $\hat{m}_{\max }$, and $\cup p \in\{2,3, \ldots, N\}$ denotes the union of $p$ sub-systems assigned with identical priority. For any $0<p_{b}<1$, the collision probability is lower compared to (11) by at least $\left(1-p_{b}\right) p_{b}^{N-1}$ due to additional randomization. Note that a node $i$ with assigned priority 0 , i.e. if $g_{i}\left(e_{k}^{i}\right)<\lambda_{i}$, can transmit only if there exists no other node with higher priority. Recalling that $g_{i}(\cdot)$ is continuous, and $e_{k}^{i}$ is a continuous Gaussian random variable for all $i \in\{1, \ldots, N\}$, it follows that $g_{i}\left(e_{k}^{i}\right)$ is also a continuous random variable. Let the cumulative distribution function, and expected value of $g_{i}\left(e_{k}^{i}\right)$ be respectively denoted by $F_{g_{i}\left(e_{k}^{i}\right)}$ and $\bar{\mu}_{g_{i}\left(e_{k}^{i}\right)}$. In addition, $\left\lceil g_{i}\left(e_{k}^{i}\right)\right\rceil$ is a discrete random variable, with the probability mass function denoted by $f_{\left\lceil g_{i}\left(e_{k}^{i}\right)\right\rceil}$. Employing the inclusion-exclusion principle and Markov's inequality, we can find the following upper-bound for the probability of collision at one time-step $k$ as

$$
\begin{aligned}
& \mathrm{P}\left[\theta_{k}^{i}=0, \forall i\right] \leq \prod_{i=1}^{N} F_{g_{i}\left(e_{k}^{i}\right)}\left(\lambda_{i}\right) \\
& +\sum_{p=2}^{N}(-1)^{p}\left(\begin{array}{c}
N \\
p
\end{array}\right)\left[\prod_{q=1}^{p} f_{\left\lceil g_{q}\left(e_{k}^{q}\right)\right\rceil}\left(\bar{m}_{k}\right)\right] \prod_{l=1}^{N-p} F_{g_{l}\left(e_{k}^{l}\right)}\left(\bar{m}_{k}\right) \\
& +\sum_{p=2}^{N}(-1)^{p}\left(\begin{array}{c}
N \\
p
\end{array}\right) \frac{\prod_{q=1}^{p} \bar{\mu}_{g_{q}\left(e_{k}^{q}\right)}}{\hat{m}_{\max }^{p}}
\end{aligned}
$$

where, $\hat{m}_{\max }=2^{n}-1$, and $\left(\begin{array}{c}N \\ p\end{array}\right)$ is the $p$-combination of the set of $N$ nodes. As expected, (12) indicates that higher number of resolution slots $n$, which results in higher number of distinct priority indexes, leads to lower collision rate.

\section{STABILITY ANALYSIS}

In this section, we study stability of the described multipleloop NCS under prioritized random access scheduling given in (10). Due to the existence of additive stochastic noise to system dynamics, we employ concepts of stochastic stability to investigate the asymptotic properties of the NCS. In addition, due to the decentralized nature of the medium access control, there exist non-zero probabilities of collisions at every single time step, and hence it is theoretically probable that collisions occur successively at all sampling times. This scenario leads to instability of the overall NCS in terms of Lyapunov $m^{\text {th }}$-mean if open-loop unstable sub-systems exist. To that end, we employ the concept of Lyapunov stability in probability (LSP), defined in the following.

Definition 1 ( [20]): An LTI system with discrete state vector $x_{k}$ is called Lyapunov stable in probability if for given $\varepsilon_{i}, \varepsilon_{i}^{\prime}>0$, there exists $\rho_{i}\left(\varepsilon_{i}, \varepsilon_{i}^{\prime}\right)>0$ such that $\left|x_{0}\right|<\rho_{i}$ implies

$$
\lim _{k \rightarrow \infty} \sup \mathrm{P}\left[x_{k}^{\top} x_{k} \geq \varepsilon_{i}^{\prime}\right] \leq \varepsilon_{i}
$$

Recall that the aggregate state of sub-system $i$ in presence of the communication constraints is $\left[x_{k}^{i^{\top}} e_{k}^{i^{\top}}\right]^{\top}$. In addition, we discussed that, in the absence of communication constraints, stabilizing gains $L_{i}$ exist such that $\left(A_{i}-B_{i} L_{i}\right)$ is stable. Therefore, having the independence of the error state $e_{k}^{i}$ from the system state $x_{k}^{i}$, we solely investigate the convergence properties of the error state $e_{k}^{i}$ in order to show stability of sub-system $i$. This is summarized in the following lemma:

Lemma 1: For a control loop $i$ with state vector $\left[x_{k}^{i^{\top}} e_{k}^{i^{\top}}\right]^{\top}$, described in (5)-(6), the system is Lyapunov stable in probability if $\xi_{i}^{\prime}>0$ and $0 \leq \xi_{i} \leq \varepsilon_{i}$ exists such that

$\lim _{k \rightarrow \infty} \sup \mathrm{P}\left[e_{k}^{i^{\top}} e_{k}^{i} \geq \xi_{i}^{\prime}\right] \leq \xi_{i}$.
Proof: $\quad$ See [11].

We define the overall NCS state as $\left[x_{k}^{\top} e_{k}^{\top}\right]^{\top}$, in which $x_{k}$ and $e_{k}$ contain local system states $x_{k}^{i}$ and local error states $e_{k}^{i}$ from all sub-systems $i \in\{1, \ldots, N\}$. Note that within a local sub-system $i$, the control and scheduling laws generate the input signals for the local system state $x_{k}^{i}$ and error state $e_{k}^{i}$ independently form the other sub-systems $j \in\{1, \ldots, N\} \backslash$ $\{i\}$. This is due to the emulation-based control law and random $g_{i}(\cdot)$ functions. Thus, local stability of all subsystems in the NCS guarantees stability of the overall NCS with the augmented state $\left[x_{k}^{\top} e_{k}^{\top}\right]^{\top}$. In terms of Lyapunov stability in probability, it translates to existence of $\xi, \xi^{\prime}>0$ such that if $\lim _{k \rightarrow \infty} \sup \mathrm{P}\left[e_{k}^{\top} e_{k} \geq \xi^{\prime}\right] \leq \xi$ holds, then exist $\varepsilon, \varepsilon^{\prime}>0$ such that $\lim _{k \rightarrow \infty} \sup \mathrm{P}\left[x_{k+1}^{\top} x_{k+1} \geq \varepsilon^{\prime}\right] \leq \varepsilon$ holds. Therefore, the LSP condition for the overall NCS becomes

$$
\lim _{k \rightarrow \infty} \sup \mathrm{P}\left[e_{k}^{\top} e_{k} \geq \xi^{\prime}\right] \leq \xi \text {. }
$$

Employing the Markov's inequality for the non-negative random variable $e_{k}^{\top} e_{k}$, we have

$$
\mathrm{P}\left[e_{k}^{\top} e_{k} \geq \xi^{\prime}\right] \leq \frac{\mathrm{E}\left[e_{k}^{\top} e_{k}\right]}{\xi^{\prime}}=\frac{\sum_{i=1}^{N} \mathrm{E}\left[\left\|e_{k}^{i}\right\|_{2}^{2}\right]}{\xi^{\prime}},
$$

which indicates that the boundedness of $\mathrm{E}\left[e_{k}^{\top} e_{k}\right]$ is sufficient for guaranteeing LSP for the overall system. The following theorem on stability of the overall systems employs this fact.

Theorem 1: Assume a multiple-loop NCS consisting of $N$ heterogeneous LTI stochastic sub-systems modeled by (1), sharing a communication channel subject to the constraint (7). Under the estimation and control laws (2) and (3), and random access prioritization law (10), with $\lambda_{i} \geq 0$, continuous and strictly increasing function $g_{i}$ 's, $p_{b} \in[0,1)$, i.e. $\mathrm{P}\left[\delta_{k}^{i}=1\right]=1-p_{b}$, and $n \geq 2$, the overall NCS with augmented state $\left[x_{k}^{\top}, e_{k}^{\top}\right]^{\top}$ is Lyapunov stable in probability.

Proof: The detailed proof is found in the Appendix.

Remark 1: The contention resolution mechanism (10) can be tuned via the locally designed parameter $\lambda_{i}$, and the channel-related parameter $\hat{m}_{\max }$. Increasing $\lambda_{i}$ decreases the competition for channel access by assigning the lowest priority, i.e. zero, to the nodes with lower error values, hence facilitates transmission opportunity for the ones with more critical situation. Setting $\lambda_{i}$ too high on the other hand leads to more sub-systems with priorities zero, and can thus lead to collision among those nodes, especially if the number of nodes are low. Higher $\hat{m}_{\max }$ means more of the contention resolution slots $n$ are available. This leads to having higher priority levels, which reduces the collision probability. On the other hand, increasing $n$ means more of the communication time slots are utilized only for prioritization and not transmission, so the trade-off should be maintained carefully. 


\section{NUMERICAL RESULTS}

We consider a multi-loop NCS comprised of $N$ stochastic sub-systems, that are divided into two homogeneous classes, i.e. unstable class ( denoted by I), and stable class (denoted by II), with the system, and input matrices as follows

$$
A_{I}=\left[\begin{array}{cc}
1.25 & 0 \\
0 & 1.1
\end{array}\right], A_{I I}=\left[\begin{array}{cc}
0.9 & 0 \\
0 & 0.9
\end{array}\right], B_{I}=B_{I I}=I_{2 \times 2} .
$$

Each sub-system $i$ is assumed to be affected by the additive random Gaussian noise with covariance matrix $W_{I}=W_{I I}=$ $I_{2 \times 2}$. Every sub-systems is controlled by a dead-beat control law $L_{i}=A_{i} B_{i}^{-1}$. We perform Monte Carlo simulations, and plot the averages over 30 runs with $95 \%$ confidence intervals. The number of sub-systems is varied within $N \in[2,60]$, where each class I and II contains $\frac{N}{2}$ sub-systems. We define the positive, continuous, and strictly increasing local function $g_{i}\left(e_{k}^{i}\right)$, introduced in (10), as the 2-norm of the networkinduced error $e_{k}^{i}$ for each sub-system $i$, i.e.

$$
g_{i}\left(e_{k}^{i}\right)=\left\|e_{k}^{i}\right\|_{2}, \quad \forall i \in\{1, \ldots, N\} .
$$

The number of resolution slots is set to $n=12$ (according to common values for powerline communication [16]), hence, the number of priority levels equals $\hat{m}_{\max }=4095$. Moreover, we assume $\lambda_{i}=0$, for all $i \in\{1, \ldots, N\}$.

\section{A. Performance Evaluation}

We compare the performance of our proposed protocol (denoted by PRIO in the figures) with some of the common scheduling schemes, such as TDMA, TOD, and random access with the optimal channel access probability $p_{b}=$ $\frac{1}{N}$, [19]. We consider two variations of TDMA: (1) full round-robin scheme wherein every node transmits without contention every $N^{\text {th }}$ time-step (denoted by TDMA), and (2) reduced round-robin wherein only unstable sub-systems transmit every $N / 2$ step, (denoted by TDMA(U)). To simulate the TOD scheme, we assume that, at each time-step, only the sub-system with the highest error norm transmits. Note that TOD approach has to be implemented in centralized fashion, hence, we assume additional communication resources exist to communicate with the centralized scheduler. For PRIO we assume no access barring, i.e. $p_{b}=0$. To evaluate the efficiency, we define two performance metrics, average error norm $\bar{E}$, and collision rate $p_{\text {coll }}$, defined as

$$
\begin{gathered}
\bar{E}=\frac{1}{N t_{\max }} \sum_{k=0}^{t_{\max }-1} \sum_{i=1}^{N}\left\|e_{k}^{i}\right\|_{2}, \\
p_{\text {coll }}=\frac{1}{t_{\max }} \sum_{k=0}^{t_{\max }-1} 1_{\left\{\theta_{k}^{i}=0, \forall i \in\{1 \ldots N\} \mid \sum_{i=1}^{N} \delta_{k}^{i} \geq 2\right\}} .
\end{gathered}
$$

Figs. 3(a) and 3(b) illustrate the performance comparisons. From 3(a), we observe that for low number of sub-systems, up to $N=4$, PRIO achieves comparable error norm as with TDMA, TDMA(U) and TOD. For higher number of subsystems though, PRIO outperforms TDMA, achieving up to 50 times lower average error norm. Expectedly, TDMA(U) performs better than TDMA, however PRIO starts outperforming TDMA(U) for $N>20$, and the performance gap increases by increasing $N$. The centralized TOD is depicted
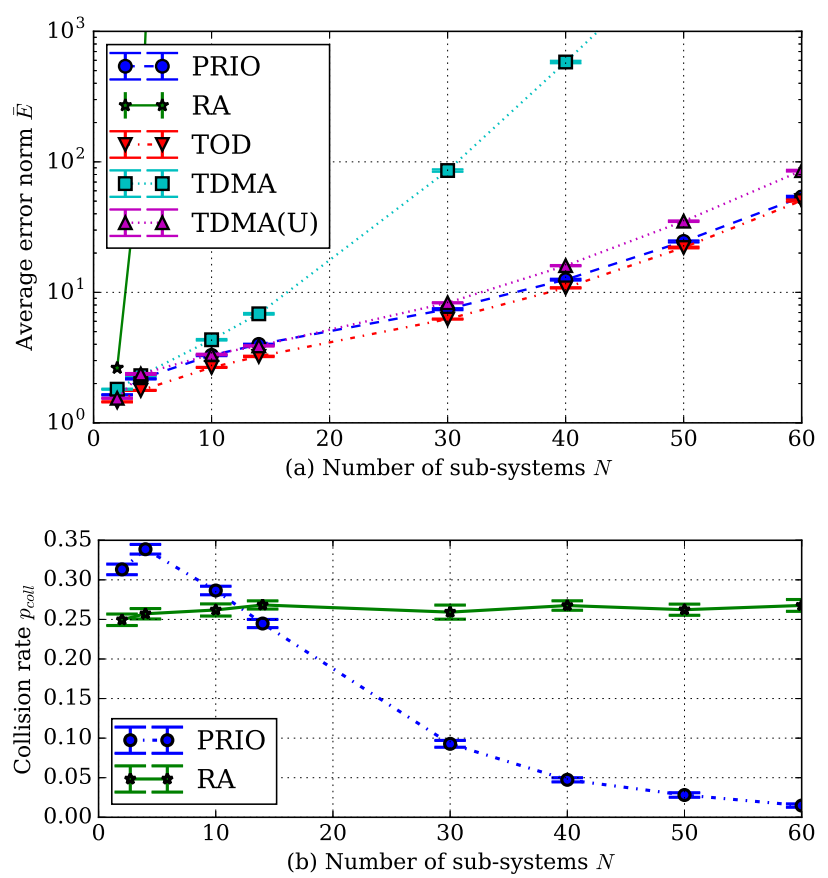

Fig. 3: (a) Average error norm $\bar{E}$ and (b) collision rate $p_{\text {coll }}$ vs. number of sub-systems $N$ for random access with back-offs (RA), prioritized contention resolution (PRIO), try-once-discard (TOD) and schedule-based access (TDMA).

here as the lower bound, achieving the best performance. Classical random access, even with optimal Bayesian backoff scheme, only delivers acceptable performance for $N=2$.

Fig. 3(b) shows that the collision rate for the PRIO is higher than the classical random access (RA) for $N<14$. Moreover, collision rates for RA saturate at $\approx 0.26$ for large $N$, due to the optimal back-off choice $p_{b}=1 / N$. Interestingly, $p_{\text {coll }}$ for PRIO start to decline for $N>4$. This is explained by higher errors for larger number of sub-systems, and hence, higher variations in the priority levels. Recall that TOD and TDMA are contention-free scheduling protocols.

\section{B. Impact of Protocol Overhead}

In order to determine which protocol is more beneficial to be employed, we suggest a joint control/communication metric $J$, which incorporates the error of each local control system, and the so called cost of the protocol [21], as

$$
J=\frac{1}{N t_{\max }} \sum_{k=0}^{t_{\max }-1} \sum_{i=1}^{N}\left\|e_{k}^{i}\right\|_{2}\left(1+\alpha_{k}^{i}\right) .
$$

where, $\alpha_{k}^{i}$ denotes the relative overhead ("cost") of the protocol. For TOD, it is defined as the ratio of resources needed to implement the centralized decision, i.e., to deliver the error information from every sub-system at every time-step to the central coordinator. Assuming that the transmission of $\left\|e_{k}^{i}\right\|$ takes time $T_{o}^{i}$, we define $\alpha_{k}^{i}=T_{o}^{i} / T_{s}$. Similarly, for PRIO protocol, overhead is defined with respect to resources consumed by the contention resolution slots, i.e. $\alpha_{k}^{i}=\left(n T_{\mathrm{CR}}\right) /\left(N T_{s}\right) \forall i, k$. We divide here by $N$, because the slots are used by all sub-systems equally. For TDMA and RA with optimal back-off, $\alpha_{k}^{i}=0$ (we assume that the back-off dimensioning and TDMA schedule allocation is done off-line, and neglect its overhead). Intuitively, the 


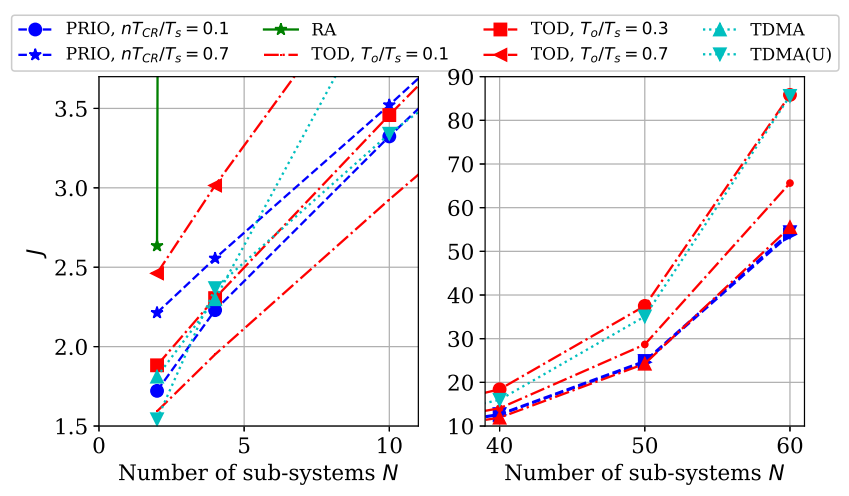

Fig. 4: Comparison of $J$ vs. number of sub-systems $N$ for different scheduling schemes: RA with back-offs, PRIO, TOD, and TDMA.

term $\left\|e_{k}^{i}\right\|_{2}\left(1+\alpha_{k}^{i}\right)$ denotes the error weighted by the cost of the protocol at a given time-step. Having $\alpha_{k}^{i}$ defined, and assuming that TOD overhead is homogeneous among subsystems, i.e., $T_{o}^{i}=T_{o} \forall i \in[1, N]$, we obtain:

$$
\begin{aligned}
J^{\mathrm{RA}}=J^{\mathrm{TDMA}} & =\frac{1}{N t_{\max }} \sum_{k=0}^{t_{\max }-1} \sum_{i=1}^{N}\left\|e_{k}^{i}\right\|_{2}, \\
J^{\mathrm{TOD}} & =\frac{T_{s}+T_{o}}{N T_{s} t_{\max }} \sum_{k=0}^{t_{\max }-1} \sum_{i=1}^{N}\left\|e_{k}^{i}\right\|_{2}, \\
J^{\mathrm{PCR}} & =\frac{N T_{s}+n T_{\mathrm{CR}}}{N^{2} T_{s} t_{\max }} \sum_{k=0}^{t_{\max }-1} \sum_{i=1}^{N}\left\|e_{k}^{i}\right\|_{2} .
\end{aligned}
$$

Fig. 4 depicts the values of $J$ for $N \in\{2,4,10,14,20\}$, and overhead values for TOD, where $T_{o} / T_{s} \in\{0.1,0.3,0.7\}$ and priority-based protocol $n T_{\mathrm{CR}} / T_{s} \in\{0.1,0.7\}$. We first observe that despite no overhead, TDMA only performs well until $N \leq 10$, and RA is only suitable for $N=2$. TDMA(U) is preferable compared to TOD with $T_{o} / T_{s}=0.7$, even for large $N$. The overhead of PRIO has a distinct effect on $J$ only for low number of sub-systems, and it scales gently with increasing $N$. Intuitively, adding more sub-systems does not increase the number of contention resolution slots $n$, hence, the overhead per sub-system decreases. On the contrary, overhead of TOD is increasing linearly with $N$. As a consequence, for $T_{o} / T_{s} \geq 0.3$ (30\% overhead for centralized decision taking) and $N>10$, PRIO results in lower joint cost than TOD, hence, is preferable to use.

\section{CONCLUSION}

In this paper we propose a state-dependent contention resolution mechanism for multi-loop NCSs with random access medium such as ad-hoc, bus or powerline networks. Introducing an error dependent measure, the priorities, ranging from 0 to a maximum priority index $\hat{m}_{\max }$, are deterministically assigned to each node at every time-step, and the highest priority, which is not necessarily $\hat{m}_{\max }$, transmits. In case more than one node is assigned with the highest priority, a collision occurs. It is shown that the overall NCS is Lyapunov stable in probability, under the proposed contention resolution mechanism. Simulation results illustrate stability claim and demonstrate noticeable performance improvement by the proposed approach compared to other common protocols. Moreover, our approach performs closely, in terms of the average error variance, to the centralized TOD approach.

\section{APPENDIX}

Proof: (Theorem 1) We prove Theorem 1 considering the worst case collision scenario which is the case under $p_{b}=0$. For any constant $1>p_{b}>0$, the obtained results can be readily repeated leading to lower collision probability, and consequently lower error expectation. To account for all the possible scenarios, we evaluate the dynamics of the error state $e_{k}$ first by assuming that there exists an interval of finite-length $k_{f} \geq N$ on the entire time axis $k \geq 0$, over which $N$ successful transmissions occur. Complementarily, we consider the scenario within which infinitely many collisions occur, such that no finite-length interval exist over which $N$ successful transmission occur. For the first scenario, we divide the entire state-space of possible error evolutions over a finite length time interval starting from an arbitrary time $k$, i.e., $\left[k, k+k_{f}\right]$, into three complementary and possible mutually exclusive cases $c_{1}, c_{2}$, and $c_{3}$, as follows:

$c_{1}$ a sub-system $i$ is assigned, at least once, the priority $\hat{m}_{k+s}^{i}=0$, at any arbitrary time-step $k+s \in\left[k, k+k_{f}\right]$, $c_{2}$ a sub-system $i$ is never assigned the priority $\hat{m}_{k+s}^{i}=$ 0 , and has successfully transmitted at least once at an arbitrary time-step $k+s \in\left[k, k+k_{f}\right]$,

$c_{3}$ a sub-system $i$ is never assigned the priority $\hat{m}_{k+s}^{i}=0$, and has never successfully transmitted over $\left[k, k+k_{f}\right]$. To exploit (16), we compute the expectation of local error norms at the time-step $k+k_{f}$, case-by-case. Recall that both functions $g_{i}\left(e_{k}^{i}\right)$ and $\left\|e_{k}^{i}\right\|_{2}^{2}$ are non-negative real-valued, continuous, and strictly increasing w.r.t. increasing the $\mathcal{L}_{1}$ norm of the error vector. According to the boundedness theorem, then $g_{i}\left(e_{k}^{i}\right)<\lambda_{i}$ implies $e_{k}^{i}$ to have bounded elements, and ensures $\left\|e_{k}^{i}\right\|_{2}^{2}<\eta_{i}$, where $\eta_{i} \in \mathbb{R}^{+}$is finite.

Assume that a sub-system $i \in c_{1}$ is assigned with priority $\hat{m}_{k+s}^{i}=0$. Hence, $g_{i}\left(e_{k+s}^{i}\right)<\lambda_{i}$, according to (10), and $\left\|e_{k+s}^{i}\right\|_{2}^{2}<\eta_{i}$. It can also be shown that $e_{k+k_{f}}^{i}$ is correlated to a past error state $e_{k+s}^{i}$, with $s \in\left[0, k_{f}-1\right]$, as follows:

$$
\begin{aligned}
e_{k+k_{f}}^{i} & =\prod_{j=s}^{k_{f}-1}\left(1-\theta_{k+j}^{i}\right) A_{i}^{k_{f}-s} e_{k+s}^{i} \\
& +\sum_{r=s}^{k_{f}-1}\left[\prod_{j=r+1}^{k_{f}-1}\left(1-\theta_{k+j}^{i}\right) A_{i}^{k_{f}-r-1} w_{k+r}^{i}\right] .
\end{aligned}
$$

The noise realizations $w_{k+r}^{i}$ are i.i.d. random variables for all $r \in\left[s, k_{f}-1\right]$, and they are also independent from $e_{k+s}^{i}$. Employing the Cauchy-Schwarz inequality, we obtain from (21)

$$
\begin{aligned}
\sum_{i \in c_{1}} \mathrm{E}\left[\left\|e_{k+k_{f}}^{i}\right\|_{2}^{2} \mid e_{k}^{i}\right] & \leq \sum_{c_{1}} \eta_{i}\left\|A_{i}^{k_{f}-s}\right\|_{2}^{2} \\
& +\sum_{c_{1}} \sum_{r=s}^{k_{f}-1} \operatorname{tr}\left(W_{i}\right)\left\|A_{i}^{k_{f}-r-1}\right\|_{2}^{2} .
\end{aligned}
$$

The LSP condition (15) can therefore be fulfilled by setting $\xi_{c_{1}}^{\prime}>\sum_{c_{1}} \eta_{i}\left\|A_{i}^{k_{f}-s}\right\|_{2}^{2}+\sum_{c_{1}} \sum_{r=s}^{k_{f}-1} \operatorname{tr}\left(W_{i}\right)\left\|A_{i}^{k_{f}-r-1}\right\|_{2}^{2}$, and, $\xi_{c_{1}}=\frac{\sum_{i \in c_{1}} \mathrm{E}\left[\left\|e_{k+k_{f}}^{i}\right\|_{2}^{2} \mid e_{k}^{i}\right]}{\xi_{c_{1}}^{\prime}}<1$.

A sub-system $i \in c_{2}$ successfully transmits at time $k+s$, then $\theta_{k+s-1}^{i}=1$, and $e_{k+s}^{i}=w_{k+s-1}^{i}$. From (21), it is then straightforward to derive the following upper-bound

$$
\sum_{i \in c_{2}} \mathrm{E}\left[\left\|e_{k+k_{f}}^{i}\right\|_{2}^{2} \mid e_{k}^{i}\right] \leq \sum_{c_{2}} \sum_{r=s}^{k_{f}} \operatorname{tr}\left(W_{i}\right)\left\|A_{i}^{k_{f}-r}\right\|_{2}^{2}
$$


The LSP condition (15) then holds by setting $\xi_{c_{2}}^{\prime}>$ $\sum_{c_{2}} \sum_{r=s}^{k_{f}} \operatorname{tr}\left(W_{i}\right)\left\|A_{i}^{k_{f}-r}\right\|_{2}^{2}$, and $\xi_{c_{2}}=\frac{\sum_{c_{2}} \mathrm{E}\left[\left\|e_{k+k_{f}}^{i}\right\|_{2}^{2} \mid e_{k}^{i}\right]}{\xi_{c_{2}}^{\prime}}<1$.

Assuming that $N$ successful transmissions occur over the finite interval $\left[k, k+k_{f}\right]$ implies that if a sub-system $i \in c_{3}$ does not transmit at $k_{f}$, then there exists a sub-system $q \notin c_{3}$ which has successfully transmitted more than once over the interval $\left[k, k+k_{f}\right]$, which the latest one occurs at time-step $k+k_{f}$. Assume that the sub-system $q$ had earlier transmitted at some time $k+s$, with $s \in\left[0, k_{f}-1\right]$. Since $\theta_{k+s-1}^{q}=1$, we have $e_{k+s}^{q}=w_{k+s-1}^{q}$. From (21), we have

$$
\mathrm{E}\left[\left\|e_{k+k_{f}-1}^{q}\right\|_{2}^{2} \mid e_{k}^{q}\right] \leq \sum_{r=s}^{k_{f}-1} \operatorname{tr}\left(W_{q}\right)\left\|A_{q}^{k_{f}-r-1}\right\|_{2}^{2} .
$$

Transmission of $q$ at time $k+k_{f}$ ahead of sub-system $i \in c_{3}$ implies $m_{k+k_{f}-1}^{q}>m_{k+k_{f}-1}^{i}$, or according to (10), $g_{q}\left(e_{k+k_{f}-1}^{q}\right)>g_{i}\left(e_{k+k_{f}-1}^{i}\right)$. Since $g_{q}(\cdot)$ is strictly increasing, we conclude $\left\|e_{k+k_{f}-1}^{q}\right\|_{2}^{2}>\left\|e_{k+k_{f}-1}^{i}\right\|_{2}^{2}$. From (24), it follows that $\mathrm{E}\left[\left\|e_{k+k_{f}-1}^{i}\right\|_{2}^{2}\right]<\sum_{r=s}^{k_{f}-1} \operatorname{tr}\left(W_{q}\right)\left\|A_{q}^{k_{f}-r-1}\right\|_{2}^{2}$. Therefore, we obtain the following for sub-system $i \in c_{3}$ :

$$
\begin{aligned}
& \sum_{i \in c_{3}} \mathrm{E}\left[\left\|e_{k+k_{f}}^{i}\right\|_{2}^{2} \mid e_{k}^{i}\right] \leq \\
& \sum_{c_{3}}\left\|A_{i}\right\|_{2}^{2} \sum_{r=s}^{k_{f}-1} \operatorname{tr}\left(W_{q}\right)\left\|A_{q}^{k_{f}-r-1}\right\|_{2}^{2}+\operatorname{tr}\left(W_{i}\right),
\end{aligned}
$$

Setting $\xi_{c_{3}}^{\prime}>\sum_{c_{3}}\left\|A_{i}\right\|_{2}^{2} \sum_{r=s}^{k_{f}-1} \operatorname{tr}\left(W_{q}\right)\left\|A_{q}^{k_{f}-r-1}\right\|_{2}^{2}+$ $\operatorname{tr}\left(W_{i}\right)$, with $\xi_{c_{3}}=\frac{\sum_{i \in c_{3}} \mathrm{E}\left[\left\|e_{k+k_{f}}^{i}\right\|_{2}^{2} \mid e_{k}^{i}\right]}{\xi_{c_{3}}^{\prime}}<1$ satisfies the LSP condition (15). This completes the proof assuming that a time interval $\left[k, k+k_{f}\right]$ can be found over that interval $N$ transmissions are successfully accomplished. Now we consider the second scenario within which no finite-length interval entailing $N$ successful transmissions over $[0, \infty)$ exists, which concludes that infinitely many collisions have occurred. Remind the upper-bound (12) for collision probability at one time-step $k$, under the prioritization law (10). We extend (12) to calculate the probability that infinitely many collisions occur over the entire time axis $[0, \infty)$, i.e.,

$$
\mathrm{P}\left[\theta_{\bar{k}}^{i}=0, \forall i \in\{1, \ldots, N\}, \forall \bar{k} \in[0, \infty)\right] .
$$

The above expression represents the probability that every transmission attempt fails at all time-steps $\bar{k} \in[0, \infty)$. From (21), if no transmission follows over $\left[k, k+k_{f}\right]$, we select $\xi^{\prime}=\sum_{i=1}^{N}\left\|A_{i}^{k_{f}} e_{k}^{i}+\sum_{r=1}^{k_{f}} A_{i}^{k_{f}-r} w_{k+r-1}^{i}\right\|_{2}^{2}$. According to (12) and (26), we can derive the following upper-bound for the probability that the error state is not Lyapunov stable:

$$
\begin{aligned}
& \sup _{e_{k}} \mathrm{P}\left[\sum_{i=1}^{N}\left\|e_{k+k_{f}}^{i}\right\|_{2}^{2} \geq \xi^{\prime}\right]<\prod_{\bar{k}=k}^{k+k_{f}} \prod_{i=1}^{N} F_{g_{i}\left(e_{\bar{k}}^{i}\right)}\left(\lambda_{i}\right) \\
& +\prod_{\bar{k}=k}^{k+k_{f}} \sum_{p=2}^{N}(-1)^{p}\left(\begin{array}{c}
N \\
p
\end{array}\right)\left[\prod_{q=1}^{p} f_{\left\lceil g_{q}\left(e_{\bar{k}}^{q}\right)\right\rceil}\left(\bar{m}_{\bar{k}}\right)\right]_{l=1}^{N-p} F_{g_{l}\left(e_{\bar{k}}^{l}\right)}\left(\bar{m}_{\bar{k}}\right) \\
& +\prod_{\bar{k}=k}^{k+k_{f}} \sum_{p=2}^{N}(-1)^{p}\left(\begin{array}{c}
N \\
p
\end{array}\right) \frac{\prod_{q=1}^{p} \bar{\mu}_{g_{q}\left(e_{\bar{k}}^{q}\right)}}{\hat{m}_{\max }^{p}} .
\end{aligned}
$$

Summing up the upper-bounds (22), (23), and (25), together with the upper-bound (26), we can find $\xi, \xi^{\prime}>0$ such that the described NCS is Lyapunov stable in probability according to the expression (15), and the proof then readily follows.

\section{ACKNOWLEDGMENT}

This work is partly funded by the German Research Foundation (DFG) within the Priority Program SPP 1914 "Cyber-Physical Networking".

\section{REFERENCES}

[1] J. P. Hespanha, P. Naghshtabrizi, and Y. Xu, "A survey of recent results in networked control systems," IEEE Proceedings, vol. 95, no. 1, 2007.

[2] G. C. Walsh and H. Ye, "Scheduling of networked control systems," Control Systems, IEEE, vol. 21, no. 1, pp. 57-65, 2001.

[3] D. Dimarogonas, E. Frazzoli, and K. Johansson, "Distributed eventtriggered control for multi-agent systems," Automatic Control, IEEE Trans. on, vol. 57, pp. $1291-1297$, may 2012.

[4] M. Rabi, G. Moustakides, and J. Baras, "Adpative sampling for linear state estimation," SIAM journal on control and optimization, 2009.

[5] A. Molin and S. Hirche, "Price-based adaptive scheduling in multiloop control systems with resource constraints," IEEE Trans. on Automatic Control, vol. 59, no. 12, pp. 3282-3295, 2014

[6] M. Vilgelm, M. Mamduhi, W. Kellerer, and S. Hirche, "Adaptive decentralized MAC for event-triggered networked control systems," in 19th Int. Conf. on Hybrid Systems: Computation and Control, 2016.

[7] G. C. Walsh, H. Ye, and L. G. Bushnell, "Stability analysis of networked control systems," IEEE Trans. on Control Systems Technology, vol. 10, no. 3, pp. 438-446, 2002.

[8] M. Mamduhi, A. Molin, D. Tolic, and S. Hirche, "Error-dependent data scheduling in resource-aware multi-loop networked control systems," Automatica, no. 81, pp. 209-216, 2017.

[9] M. Mamduhi, A. Molin, and S. Hirche, "Event-based scheduling of multi-loop stochastic systems over shared communication channels," in 21st Int. Symp. on Mathematical Theory of Networks and Systems, pp. 266-273, 2014.

[10] M. Donkers, W. Heemels, D. Bernardini, A. Bemporad, and V. Shneer, "Stability analysis of stochastic networked control systems," Automatica, vol. 48, no. 5, pp. 917-925, 2012.

[11] M. Mamduhi, M. Kneissl, and S. Hirche, "Decentralized eventtriggered medium access control for networked control systems," in 55th IEEE Annual Conf. on Decision and Control, pp. 513-519, 2016.

[12] A. Laya, L. Alonso, and J. Alonso-Zarate, "Is the Random Access Channel of LTE and LTE-A Suitable for M2M Communications? A Survey of Alternatives," IEEE Commun. Surveys \& Tut., vol. 16, no. 1, pp. 4-16, 2014.

[13] K. Gatsis, A. Ribeiro, and G. J. Pappas, "Control-aware random access communication," in Cyber-Physical Systems (ICCPS), 2016 ACM/IEEE 7th International Conference on, pp. 1-9, IEEE, 2016.

[14] M. Vilgelm, H. M. Gürsu, W. Kellerer, and M. Reisslein, "LATMAPA Load-Adaptive Throughput-Maximizing Preamble Allocation for Prioritization in 5G Random Access," IEEE Access, vol. 5, pp. 1103 1116,2017

[15] M. Vilgelm, S. Rueda Liñares, and W. Kellerer, "Enhancing Cellular M2M Random Access with Binary Countdown Contention Resolution," in IEEE International Symposium on Personal, Indoor and Mobile Radio Communications (PIMRC), Oct 2017.

[16] T. Gehrsitz, R. Durner, H. Kellermann, H. T. Lim, and W. Kellerer, "Priority-based energy-efficient MAC protocols for the in-car power line communication," in 2014 IEEE Vehicular Networking Conference (VNC), pp. 61-68, Dec 2014.

[17] M. Mamduhi, D. Tolic, A. Molin, and S. Hirche, "Event-triggered scheduling for stochastic multi-loop networked control systems with packet dropouts," in 53rd IEEE Conf. on Decision and Control, pp. 2776-2782, 2014.

[18] T. You, C. Yeh, and H. Hassanein, "A new class of collision prevention MAC protocols for wireless ad hoc networks," in IEEE International Conf. on Communications, vol. 2, pp. 1135-1140 vol.2, 2003.

[19] R. Rivest, "Network control by bayesian broadcast," IEEE Transactions on Information Theory, vol. 33, pp. 323-328, May 1987.

[20] F. Kozin, "A survey of stability of stochastic systems," Automatica, vol. 5, pp. 95-112, Jan. 1969.

[21] A. Tanenbaum and D. Wetherall, Computer Networks. Pearson Prentice Hall, 2011. 